\title{
CORRECTION
}

\section{Correction: Quality of life under extended continuous versus intermittent adjuvant letrozole in lymph node-positive, early breast cancer patients: the SOLE randomised phase 3 trial}

Karin Ribi, Weixiu Luo, Marco Colleoni, Per Karlsson, Jacquie Chirgwin, Stefan Aebi, Guy Jerusalem, Patrick Neven, Vincenzo Di Lauro, Henry L. Gomez, Thomas Ruhstaller, Ehtesham Abdi, Laura Biganzoli, Bettina Müller, Annelore Barbeaux, Marie-Pascale Graas, Manuela Rabaglio, Prudence A. Francis, Theodoros Foukakis, Olivia Pagani, Claudio Graiff, Daniel Vorobiof, Rudolf Maibach, Angelo Di Leo, Richard D. Gelber, Aron Goldhirsch, Alan S. Coates, Meredith M. Regan and Jürg Bernhard; on behalf of the SOLE Investigators

British Journal of Cancer (2020) 122:1119; https://doi.org/10.1038/s41416-019-0709-x

Correction to: British Journal of Cancer (2019) 120, 959-967; https://doi.org/10.1038/s41416-019-0435-4; published online 10 April 2019.
The ClinicalTrials.gov Identifier was incorrectly listed as NCT00651456. The correct identifier is listed below.

Clinical trial information

Clinical trial information: NCT00553410. 